\title{
Maldoror ou la méchanceté sublimée
}

\author{
Jonathan Petitot \\ Université d'Aix-Marseille
}

«Lautréamont, prophète de la poésie libérée, celui qui a montré que la littérature pouvait encore essayer d'exprimer l'homme dans sa totalité. » (Le Clézio, 1973, p. 9 ; préface aux Chants de Maldoror. Isidore Ducasse, qui publie en 1869 Les Chants de Maldoror sous le pseudonyme de Comte de Lautréamont, demeure une énigme tant sur le plan biographique que sur le plan littéraire ; mais la renommée de l'œuvre, elle, n'est plus à faire. La première lecture de ces pages est toujours une violence; qu'ils entrainent une admiration forcenée ou un dégoût véhément, Les Chants de Maldoror restent une œuvre scandaleuse, marquée par le sceau du génie ou par celui de 
l'opprobre. Toute la dimension scandaleuse de ce morceau de bravoure poétique gravite justement autour du concept de méchanceté : s'il est un adjectif qu'une première lecture donnerait volontiers au personnage de Maldoror, c'est bien celui de "méchant», comme la première syllabe de son nom le laisse déjà signifier; car ce héros - s'il est encore permis d'employer ce terme, abstraction faite de son ancienne dimension morale et considérant le fait que tous les codes de la narratologie sont foulés aux pieds - semble, au cours de ses pérégrinations, chercher à s'enfoncer le plus loin possible dans la carrière du mal. Toutefois, si la méchanceté est au cœur de chaque page, c'est avant tout sur le mode de l'interrogation : est-elle plaisamment délectable ou vainement récréative, proprement humaine ou sauvagement animale, égoïstement contingente ou divinement nécessaire ? Ainsi Maldoror est-il le parfait reflet de son époque, héritier du questionnement romantique sur la morale établie, précurseur de la révolte surréaliste ; ses exactions soulèvent bien des interrogations, car elles sont des cris poussés qui interpellent et questionnent sur le plan moral et le plan humain.

Il reste à déterminer quel(s) sens donner à ces actions, car si un premier degré de lecture fait de Maldoror un héraut du mal, le chantre de la méchanceté au sein d'une œuvre dont les pages pourraient constituer une typographie complète de la méchanceté, la découverte progressive du personnage amène à discerner un sentiment de révolte qui pousse le personnage à transcender le manichéisme moral biblique, omniprésent dans l'œuvre. 


\section{Un héraut du mal}

Quelle que soit la part biographique de l'entreprise ducassienne, les choix amoraux ou immoraux qui constituent l'esthétique de l'œuvre sont le fruit d'une lignée littéraire florissante. Dans une lettre au codirecteur d'une grande maison d'édition internationale, Lautréamont se déclare le successeur, entre autres, de John Milton ou de Charles Baudelaire ${ }^{1}$.

Si la carrière du mal a en effet déjà été explorée sur le plan esthétique, Ducasse entend lui donner une vigueur innovante et une profondeur nouvellement savoureuse, afin de concevoir ce qu'il nomme dans son incipit des «landes inexplorées ». À Milton, il emprunte l'esprit de révolte de son personnage, son questionnement permanent, l'amour romantique d'une liberté à conquérir, la volonté de ne plier devant personne, quel qu'en soit le prix. À Baudelaire, il doit cette délectation devant le Mal comme objet poétique, il doit ses métaphores triviales mais signifiantes, il doit la puissance de son langage et de ses symboles. Mais s'il y a quelque chose qu'il ne doit qu'à lui-même, c'est la verbalisation poétique de la violence; l'esthétisation de la cruauté a déjà été entreprise, comme il le souligne lui-même, mais l'innovation ducassienne repose dans la richesse évocatrice de l'image poétique et de sa manifestation lexicale et stylistique. Chez Lautréamont, et c'est ce qui rend son œuvre unique, se mêlent une délectation déconcertante pour l'acte méchant et un travail de la forme et de l'expressivité verbale peu commun. Ouvrir Les Chants de

\footnotetext{
1 Lettre à Verboeckhoven, Paris 23 octobre : «J'ai chanté le mal comme ont fait Mickiéwickz, Byron, Milton, Southey, A. de Musset, Baudelaire, etc. Naturellement, j'ai un peu exagéré le diapason pour faire du nouveau dans le sens de cette littérature sublime qui ne chante le désespoir que pour opprimer le lecteur. » (Ducasse, 1973, p. 271)
} 
Maldoror, c'est trouver un travail poétique en cours de construction, c'est observer la sauvagerie être domptée par la plume; et comme tout acte de création observé sur le fait, il est authentique et véritable.

Dès les premières lignes de l'œuvre, le lecteur est averti de ce qu'il trouvera en tournant les pages. Les expressions explicites sont en effet légion : il parle des «pages sombres et pleines de poison» (I, p.17) et évoque «les émanations mortelles de ce livre » (ibid.). Cette entrée en matière, d'une franchise déconcertante, éveille nécessairement la curiosité du lecteur; dès lors, difficile de refermer le livre en se déclarant choqué par la méchanceté, car le lecteur a été dûment prévenu. Bien plus que cela : c'est justement sur la curiosité malsaine des lecteurs potentiels que joue cet incipit original. Ainsi prend-t-il l'âme trop humaine du lecteur à partie: «C'est peut-être la haine que tu veux que j'invoque.» Et l'auteur d'insister sur l'appétit légitime de ce "monstre ». Aussi la méchanceté, plus que simple objet d'étude ou sujet de scandale, plus qu'un plaisir assuré de choquer un lectorat encore bien-pensant, est un moyen de renouveler la complicité entre l'auteur et le lecteur ; quand Stendhal jouait sur le partage d'un regard ironique porté sur les personnages, Lautréamont renouvelle cette complicité, car il partage avec le lecteur sa fascination pour l'acte de cruauté tout en le contraignant à l'honnêteté dans ses motivations de lecture.

Cette aventure épique et poétique, dont les strophes se succèdent sans cohérence temporelle et diégétique discernable, est teintée d'une volonté sous-jacente de scandaliser ; certains critiques ont souligné la rancœur d'Isidore Ducasse contre sa scolarité et, en particulier, contre la classe de rhétorique. Aussi, 
une lecture sainte-beuvienne de l'œuvre - aussi restrictive soit-elle - ferait-elle de la volonté de poétisation du mal l'assouvissement d'une rancœur adolescente contre les adultes en tous genres et, en particulier, contre une société judéochrétienne embourgeoisée; il faut reconnaître, à la lecture de l'œuvre, que la volonté de révolte qui en émerge a bien une dimension adolescente, qui confinerait à une sorte de pureté dans le mal, en donnant à cette volonté de blesser un tour franc et naïf. Contrairement à certains méchants habituels et traditionnels - notamment les criminels sadiens, qui ajoutent à leurs méfaits le poids du mensonge, en donnant au vice l'apparence de la vertu, ou en parant leurs gestes de discours sophistiques et manipulateurs - Maldoror revendique ses actions dans toute leur noirceur et souligne le poids de sa volonté ; ainsi clame-t-il au lecteur, dans la treizième strophe du chant II : «Que m'importe le jugement dernier ! Ma raison ne s'envole jamais, comme je le disais pour vous tromper. Et quand je commets un crime, je sais ce que je fais : je ne voulais pas faire autre chose!» (p. 109). Si les causes de la méchanceté de Maldoror font partie des questionnements renouvelés au sein de l'œuvre, comme nous l'observerons ultérieurement, la première information, la plus éclatante, que Lautréamont nous donne est son caractère naturel. Cette méchanceté lui est inhérente, et son envie est irrépressible. Cependant, la grande question ducassienne, qui n'est pas sans rappeler le poème de Milton, est de savoir si cette nature méchante, qui le met au ban du monde des hommes, semble être condamnée par le Créateur alors qu'elle est en un sens de son fait. Car Lautréamont emploie le terme imposant de «fatalité » alors qu'il fait le portrait de son alter ego littéraire, à l'ouverture du premier chant : 
J'établirai dans quelques lignes comment Maldoror fut bon pendant ses premières années, où il vécut heureux ; c'est fait. Il s'aperçut ensuite qu'il était né méchant: fatalité extraordinaire! Il cacha son caractère tant qu'il put, pendant un grand nombre d'années; mais, à la fin, à cause de cette concentration qui ne lui était pas naturelle, chaque jour le sang lui montait à la tête ; jusqu'à ce que, ne pouvant plus supporter une pareille vie, il se jeta résolument dans la carrière du mal... atmosphère douce! (p. 19)

Le portrait impressionniste que Lautréamont donne de Maldoror, ce portrait qui se construit touche après touche, n'est pas le portrait du méchant «type». Nous sommes loin de la méchanceté purement narrative de Méléagant, faire-valoir de Lancelot; nous sommes loin également de l'autogratification paradigmatique de Valmont et de la marquise de Merteuil dans leurs entreprises de libertinage. Chez Maldoror, la méchanceté transcende le concept même de plaisir; il y a certes une douceur, une suavité de l'acte méchant, mais le bénéfice psychologique ne réside pas dans cette douceur. À la lecture des Chants, la question se pose nécessairement de la gratuité du mal, car le plaisir est fugace et quasiment ignoré par Maldoror. $\mathrm{Au}$ lieu de cela, nous découvrons progressivement un personnage insatisfait, plongé dans un mal-être constant; si les personnages d'un auteur tel que Sade sont emplis de vie par leurs méfaits, Maldoror, au contraire, ne semble pas s'en satisfaire, bien qu'il ne change pas de ligne de conduite pour autant. Ses confrères humains le perçoivent comme un être mort, désincarné. À l'ouverture du deuxième chant, Lautréamont confie au lecteur : "sur sa figure ne brillent que les reflets du cadavre » (p. 62) ; de même, le prêtre qui officie aux funérailles d'un homme, au chant $V$, avertit ses ouailles au sujet de Maldoror : 
Vous ne semblez pas vous douter que celui-ci, que la maladie força de ne connaître que les premières phases de la vie, et que la fosse vient de recevoir dans son sein, est l'indubitable vivant; mais, sachez, au moins que celui-là, [...] quoiqu'il ait beaucoup vécu, est le seul véritable mort. (p. 217)

Cette dimension essentielle du protagoniste suggère alors que la méchanceté du personnage serait un moyen, non de se satisfaire, mais de capter une essence de vie, d'appréhender une émotion, de ressentir; un moyen de vivre. Il n'est alors guère surprenant de voir Gaston Bachelard, dans son Lautréamont, faire un parallèle entre le «vouloir-vivre » de Schopenhauer et ce qu'il appelle un «vouloir-attaquer»chez Ducasse ; la conscience de Maldoror se réalise, son sentiment de vivre s'exalte dans l'agression. Hors de la brièveté de ces moments, il est privé de vie et, à plusieurs reprises, il passe même pour la figure de la mort personnifiée. Cependant, l'esthétique et la philosophie du lautréamontisme reposent sur l'énergie et sur l'action; aussi cette vacuité existentielle n'entrave-t-elle pas le personnage, mais le pousse dans une attaque plus ardente et plus violente, qui le transforme en esprit de révolte.

\section{Maldoror, visage de la révolte}

La nature du personnage de Maldoror demeurera à dessein insaisissable; en fonction de ses pérégrinations, il semble ne pas appartenir au monde des humains, apparaissant davantage comme un esprit, voire un démon, mais parfois, et ce, dans ses pires moments, il est humain, trop humain. Cependant, il lutte en permanence contre cette nature humaine ; s'il est, à son avis, 
un plus grand mal que lui, c'est l'humanité elle-même: le leitmotiv de l'œuvre est bien que dans le règne animal conception importante dans la cosmogonie lautréamontiste ce qui distingue l'homme des autres espèces est son aptitude à la méchanceté. Très rapidement au cours des chants, la lecture morale du monde s'inverse: l'esprit Maldoror, qui incarne initialement la méchanceté pure et étrangère, devient simplement l'incarnation de la méchanceté humaine et se complaît dans le fait de renvoyer à l'humanité les méfaits qu'elle inflige. La méchanceté humaine est le fondement de l'œuvre, ce que Lautréamont présente dès le premier chant :

Moi je fais servir mon génie à peindre les délices de la cruauté ! Délices non passagères, artificielles ; mais qui ont commencé avec l'homme, finiront avec lui. [...] Dieu, qui l'a créé avec magnificence, c'est toi que j'invoque : montre-moi un homme qui soit bon! ... Mais que ta grâce décuple mes forces naturelles; car, au spectacle de ce monstre, je puis mourir d'étonnement : on meurt pour moins. (p. 20)

Si nous postulons alors que la méchanceté est ce qui caractérise l'homme, les actions de Maldoror peuvent prendre des significations supplémentaires. Être méchant deviendrait alors une sorte de comportement mimétique pour s'apparenter à l'homme, l'appréhender et tenter de le comprendre. Le geste symbolique, dans la sixième strophe du chant $\mathrm{I}$, où Maldoror boit les larmes d'un adolescent qu'il vient de torturer, montre une volonté de comprendre et de s'approprier un comportement humain, une part de la souffrance, mais aussi, par mimétisme, d'essayer d'agir en humain. Une différence est ici soulignée par Bachelard, qui illustre le fait que la méchanceté, si elle est humaine, est l'apanage des adultes, tandis que l'enfant demeure une image typique de l'innocence. Le passage à l'âge adulte est donc passage à la méchanceté, qui 
apparaît comme une voie initiatique nécessaire. Un épisode du chant II est particulièrement significatif à cet égard : dans la strophe 4, un enfant épuisé court après un omnibus pour être ramené chez lui ; il crie, il pleure, mais le conducteur et les passagers font la sourde oreille, agacés par « ces gémissements au timbre argentin ». Toutefois, un jeune homme réagit :

Seul, un jeune homme, plongé dans la rêverie, au milieu de ces personnages de pierre, paraît ressentir de la pitié pour le malheur. En faveur de l'enfant, qui croit pouvoir l'atteindre, avec ses petites jambes endolories, il n'ose pas élever la voix ; car les autres hommes lui jettent des regards de mépris et d'autorité, et il sait qu'il ne peut rien faire contre tous. Le coude appuyé sur ses genoux et la tête entre ses mains, il se demande, stupéfait, si c'est là vraiment ce qu'on appelle la charité humaine. [...] Il se démène, mais en vain, dans le siècle où il a été jeté ; il sent qu'il n'y est pas à sa place, et cependant il ne peut en sortir. [...] L'adolescent se lève, dans un mouvement d'indignation, et veut se retirer, pour ne pas participer, même involontairement, à une mauvaise action. Je lui fais un signe, et il se remet à mon côté... (p. 66-67).

À cet instant, Maldoror apparaît comme le compagnon qui assiste le rite initiatique, qui permet au jeune homme de s'intégrer à l'humanité, en confirmant sa méchanceté malgré lui. Mais si Maldoror rapporte les sentiments du jeune homme, c'est bien parce qu'il en est l'émanation la plus extrême ; luimême entretient une haine de l'humanité à laquelle il ne peut appartenir.

Face à un échec à donner une signification humaine à sa méchanceté, il n'est guère surprenant de voir le personnage de Maldoror se réfugier dans l'animalité ; les métaphores et les métamorphoses animales sont omniprésentes et rythment les différents chants. Pour Maldoror, elles sont une façon de rejeter la part humaine de son être, pour adopter une conduite sensée : 
la violence animale est moins cruelle que la méchanceté humaine ; un épisode souligne en particulier cette évolution du personnage de Maldoror: dans la treizième strophe du deuxième chant, il se tient sur le rivage et admire une tempête qui détruit un navire. Il se délecte de voir périr ses semblables : «Ô ciel! Comment peut-on vivre, après avoir éprouvé tant de voluptés! Il venait de m'être donné d'être témoin des agonies de mort de plusieurs de mes semblables.» (p.106) Cette cruauté bien humaine s'approfondit alors qu'il décide de s'armer d'un fusil et de tirer sur les rescapés qui tentent de s'accrocher aux écueils. Cependant, alors que le naufrage se poursuit, il est témoin de l'arrivée de requins qui viennent dévorer les nageurs ; il décide de se mêler à eux et s'animalise, nageant à leurs côtés, jusqu'à rencontrer une femelle requin dont il dira que c'est son premier amour. Cette épisode marque la première animalisation véritable de la méchanceté de Maldoror, méchanceté humaine et absurde qui devient peu à peu cruauté implacable mais majestueuse de la nature, qui crée chez lui un sentiment qui s'épanouit. On le voit, à la huitième strophe du premier chant, regretter son humanité :

Je suis fils de l'homme et de la femme, d'après ce qu'on m'a dit. Ça m'étonne, je croyais être davantage. Au reste que m'importe d'où je viens? Moi si cela avait pu dépendre de ma volonté, j'aurais voulu être le fils de la femelle du requin, dont la faim est amie des tempêtes, et du tigre à la cruauté reconnue ; je ne serai pas si méchant. (p. 29)

Cette métaphore est parfaitement représentative de cet élan vers l'animalité d'un Maldoror qui souhaiterait échapper à sa condition parmi les hommes. Son agression permanente contre l'humanité semble donc provenir d'un sentiment d'injustice, de révolte, contre l'ordre des choses et contre son 
Créateur; la dimension blasphématoire de l'œuvre est clairement revendiquée à la quatrième strophe du chant II :

Ma poésie ne consistera qu'à attaquer, par tous les moyens, l'homme, cette bête fauve, et le Créateur, qui n'aurait pas dû engendrer une pareille vermine. Les volumes s'entasseront sur les volumes, jusqu'à la fin de ma vie, et, cependant, l'on n'y verra que cette seule idée, toujours présente à ma conscience ! (p. 68)

Il voue à cette figure du démiurge une haine incommensurable, qui s'avère être le fil conducteur de l'œuvre. À bien des égards, le personnage de Maldoror peut revêtir un visage d'Antéchrist. Son parcours est jonché d'épisodes symboliques qui rappellent ceux du Christ, les visions de l'Apocalypse accompagnent ses apparitions, sa lutte perpétuelle contre les anges et contre Dieu, son aura effrayante qui les met à genoux, sont les nombreux éléments étayant cette théorie. Maldoror, à l'instar du diable, est un tentateur : on le voit au fil des Chants tenter de corrompre trois jeunes garçons, afin de les mener sur la voie du mal et de la méchanceté pour, semble-t-il, montrer sa puissance au créateur. Mais quelle image donne-t-il de celui qui a créé la vie, qui est censé être la bonté même? Celle d'un nouveau Lucifer dantesque, à la huitième strophe du deuxième chant :

Je soulevai la paupière effarée plus haut, plus haut encore, jusqu'à ce que j'aperçusse un trône, formé d'excréments humains et d'or, sur lequel trônait, avec un orgueil idiot, le corps recouvert d'un linceul fait avec des draps non lavés d'hôpital, celui qui s'intitule lui-même le Créateur ! Il tenait à la main le troc pourri d'un homme mort, et le portait, alternativement, des yeux au nez et du nez à la bouche ; une fois à la bouche, on devine ce qu'il en faisait. [...] Quelquefois il s'écriait : «Je vous ai créés; donc j'ai le droit de faire de vous ce que je veux. Vous ne m'avez rien fait, je ne dis pas le contraire. Je vous fais souffrir, et c'est pour mon plaisir. (p. 81) 
La situation telle que Maldoror - ou Lautréamont - la présente est particulièrement paradoxale : le Créateur apparaît sous les traits de l'horreur symbolisée. Ce travestissement impie n'a d'autre signification symbolique que celle de montrer que le démiurge est l'origine de toute cruauté et que les apparences de bonté cachent un pouvoir despotique et cruel sur les hommes; le rapport entre Créateur et créature est perverti, se transformant en un rapport de domination qui place l'homme sous le joug d'une illusion oppressive; il est intéressant de préciser que la réaction de Maldoror à la suite de cette vision horrifique sera de pousser un cri de peur si fort que son ouïe s'éveillera au monde. Cette vision plus que marquante dans l'existence du personnage est une parfaite représentation onirique de la perception que Maldoror a du monde : violente, agressive et agressée. De là vient cependant une cohérence dans le comportement permanent de Maldoror, qui explique son attitude provocatrice et sacrilège : il sera celui qui mettra fin au règne du créateur ou, du moins, celui qui sapera son influence sur les hommes afin de les affranchir et de les libérer. Cette libération prend la forme d'une méchanceté violente, éclatante et constante qui finit par devenir constitutive de sa psyché et par dominer toutes ses actions pour les orienter dans un seul but.

\section{Par-delà le bien et le mal}

Si les références à la bible et à son antagonisme manichéen entre le bien et le mal sont légion, la méchanceté de Maldoror ne s'y inscrit pas en profondeur. Comme nous l'avons vu, la 
construction morale biblique est intégralement bouleversée, à tel point que le Créateur porte le visage du diable et sa déchéance dépasse celle de son adversaire. Mais Maldoror, s'il se fait un plaisir de s'attaquer à Dieu, n'est pas pour autant un suppôt du diable. Le rapport qu'il entretient avec ce dernier est particulier : s'il pourrait apparaître comme son disciple le plus doué au début du livre, se réclamant même de son œuvre, il s'affranchit néanmoins de sa tutelle jusqu'à le repousser. Un épisode est particulièrement signifiant à cet égard : à la fin de l'œuvre, à la quatrième strophe du Chant V, Maldoror reçoit la visite d'un serpent. La lecture symbolique biblique, omniprésente dans la poésie ducassienne, confirme durant cet échange qu'il s'entretient avec Satan; or, il n'hésite pas à repousser violemment cet interlocuteur infernal : «Il n'est pas loin, le jour, où mon bras te renversera dans la poussière, empoisonnée par ta respiration, et, arrachant de tes entrailles une nuisible vie, laissera sur le chemin ton cadavre, criblé de contorsions.» (p. 205) Cette déclaration directe et virulente montre au lecteur que la haine de Maldoror pour le Créateur est également tournée vers son adversaire et que, in fine, le personnage de Maldoror rejette tout, y compris le Mal dans toute sa dimension biblique. Sa volonté de révolte se tourne vers toutes les idoles morales, qu'il s'agisse de la figure traditionnelle de la bonté, mais aussi de celle de la méchanceté poussiéreuse et dépassée que Satan représente. Sa méchanceté propre et renouvelée n'a rien à voir avec cette vision du mal, ce concept de péché, mais c'est une méchanceté personnelle, unique, romantique, un mal libéré de toute entrave.

Le personnage de Maldoror, et Isidore Ducasse avec lui, s'en prennent à l'émanation la plus sensible de la morale «conventionnelle» sur le plan psychologique : la conscience. 
Rien de moins surprenant, considérant la volonté de scandale de l'œuvre: dans leur entreprise de libération de l'homme, Les Chants de Maldoror mettent à bas toutes les chaînes qui le retiennent captif. À de nombreuses reprises dans les Chants, cette conscience est nommément et explicitement stigmatisée. Un exemple en est donné à la fin du Chant II : «Qu'il n'envoie plus sur la terre la conscience et ses tortures. J'ai enseigné aux hommes les armes avec lesquelles on peut la combattre avec avantage. Ils ne sont pas encore familiarisés avec elle; mais tu sais que, pour moi, elle est comme la paille qu'emporte le vent. » (p. 117) À savoir donc si Maldoror a une conscience, la réponse est nietzschéenne : non, car il n'en a pas besoin. Cela signifie-t-il pour autant que ce personnage évolue dans le monde sans cohérence psychologique pour guider son comportement? Assurément non. Au contraire, au fil des Chants, nous pouvons observer se construire un sens de l'éthique; une éthique déçue, pervertie, certes, mais qui n'est pas départie d'une certaine cohérence. Comme nous l'avons brièvement évoqué précédemment, la méchanceté de Maldoror est réaction avant d'être action; c'est une défense contre la cruauté humaine et divine, c'est une volonté de détruire la source de son mal-être. Dans sa défiance constante de l'homme et de toute la création et dans son combat perpétuel contre l'humain et le divin, Maldoror apparaît désorienté, désemparé face aux manifestations de bonté, qui sont des entorses à sa conception du monde et des remises en question de la haine confortable dans laquelle il s'est enfermé. Un excellent exemple en est donné à la strophe 5 du chant II ; après avoir décrit une jeune fille qui le suit régulièrement avec, sur le visage, un air de bonté qui le laisse particulièrement perplexe et mal à l'aise, Maldoror imagine lui adresser les paroles suivantes : 
Dans un moment d'égarement, je pourrais te prendre les bras, les tordre comme un linge lavé dont on exprime l'eau, ou les casser avec fracas, comme deux branches sèches, et te les faire ensuite manger, en employant la force. Je pourrais, en prenant ta tête entre mes mains, d'un air caressant et doux, enfoncer mes doigts avides dans les lobes de ton cerveau innocent, pour en extraire le sourire aux lèvres, une graisse efficace qui lave mes yeux, endoloris par l'insomnie éternelle de la vie. (p. 70)

Une réaction d'une telle violence ne semble a priori provenir que d'un malaise et d'une incompréhension face à cette figure de la bonté, qui désarme le vouloir-attaquer que Bachelard lui prête; néanmoins il y a un enjeu, une envie presque, qui souligne cette sensibilité viciée, offensée, à la bonté.

Nous pouvons également observer une certaine constance lorsque Maldoror se montre compatissant ; le fait est rare, certes, mais avéré, et à chaque occurrence, la compassion du personnage est destinée à des personnages qui sont, eux aussi, au ban de la société ; cette affection pour les réprouvés se trouve lors d'un échange shakespearien avec un fossoyeur à qui Maldoror propose de prêter la main à la fin du premier chant ; il réconforte une allégorie de la prostitution au chant 7 allégorie qui va même jusqu'à lui dire qu'il est «bon » envers elle - et il ressent une sympathie teintée d'admiration envers les prostituées, qu'il perçoit comme les «cristallisations d'une beauté morale supérieure ». Ces éléments pourraient infirmer une vision trop unilatérale, une caractérisation trop monolithique de ce personnage de Maldoror, qui gagne en complexité à chaque lecture ; ils pourraient alors souligner que, dans de rares cas où il perçoit une forme d'injustice, Maldoror n'est pas tant un monstre de cruauté profane et insensible 
qu'un être qui laisse sa méchanceté s'exprimer quand il l'estime justifié, selon une cohérence psychologique qui lui est propre.

Dans ce véritable maelström qu'est la question de la méchanceté au cœur des Chants de Maldoror, la seule affirmation indiscutable est la volonté de libération. Comme nous l'avons précédemment évoqué, l'attitude sacrilège et violente de Maldoror provient d'un mal-être existentiel, d'une impossibilité à trouver sa place dans l'ordre du monde tel qu'il est ; conscient de sa souffrance, il l'impute à la figure divine : «Dieu, je ne te retarderai pas davantage ; et pour t'instruire et te préserver, réfléchis au sort fatal qui m'a conduit à la révolte, quand peut-être j'étais né bon!» (IV, p. 170) Ainsi, l'œuvre poétique, qui n'est au premier degré constituée que des pérégrinations malfaisantes d'un personnage ô combien cruel, peut également être perçue comme le laboratoire d'une réflexion sur l'origine de l'acte méchant: Maldoror, icône de cruauté, se déclare le miroir de la méchanceté humaine, tout en blâmant Dieu pour l'avoir reçue et pour en avoir fait don à l'humanité ; plus qu'une pratique de vie, un choix permanent d'actions égoïstes et tournées vers la satisfaction, la méchanceté est dans les Chants un concept réflexif, vivant, qui évolue et change de visage, à tel point que Maldoror est amené à approfondir sa propre compréhension du mal. Ce n'est pas sans émotions que le lecteur peut observer, à la sixième strophe du premier chant, le personnage indécis se questionnant sur les fondements du système moral : «Hélas ! Qu'est-ce donc que le bien et le mal! Est-ce une même chose par laquelle nous témoignons avec rage notre impuissance, et la passion d'atteindre à l'infini par les moyens les plus insensés ? Ou bien, sont-ce deux choses différentes ?» (p. 24) Ainsi l'auteur donnet-il tôt dans l'œuvre des indices afin de comprendre Maldoror et 
ses actes; cette méchanceté ne serait-elle pas l'un de ces « moyens les plus insensés » pour «atteindre à l'infini »? Elle serait alors, non une fin satisfaisante en soi, mais un moyen de se libérer du joug de la morale, de la conscience, un moyen de crier à l'homme et à Dieu que sa place dans le monde le laisse insatisfait. Comment ne pas observer l'écho entre ces questions que nous venons de citer et cette phrase qu'il écrit dans le premier chant «Moi, comme les chiens, j'éprouve le besoin de l'infini... »? Sa conduite n'est pas le fruit du plaisir, ni d'une errance désorientée, mais bien la pulsion violente qui répond à ce besoin inassouvi de l'infini, la volonté inébranlable de résoudre l'inadéquation entre sa conception du monde et la réalité telle qu'il l'affronte. Une réalité qui se caractérise, à ses yeux, comme un monde dominé par le mensonge. Aussi sa croisade révoltée contre l'ordre établi, bien qu'elle se réalise dans une conduite ultimement répréhensible, devient-elle une lutte contre le mensonge, et sa méchanceté devient une proclamation pour la vérité humaine :

Ainsi, donc, l'hypocrisie sera chassée carrément de ma demeure. Il y aura, dans mes chants, une preuve imposante de puissance, pour mépriser ainsi les opinions reçues. [...] Libre comme la tempête, il est venu échouer, un jour, sur les plages indomptables de sa terrible volonté ! Il ne craint rien, si ce n'est lui-même! (IV, p. 160)

Par conséquent, cette attitude sacrilège contre l'homme et ses valeurs d'humanité, contre Dieu et les principes de son culte, ne sont finalement que le moyen le plus paradoxal de lutter contre l'hypocrisie et le mensonge. Ainsi le parallèle de sens entre Paradise Lost de Milton et Les Chants de Maldoror se confirme, car dans sa volonté de mal, Maldoror devient lucifer au sens latin originel de porteur de lumière, en portant aux 
hommes ses propres révélations sur la vérité de la morale, de la religion et de l'obéissance bornée et aveugle à un code de conduite qui soit contraire à leur nature authentique. À travers ses tirades sur la cruauté et ses malédictions contre Dieu et contre le démon, sa voix se veut donc celle d'une vérité bien plus fondamentale, qui rétablirait l'homme en respectant son intégrité, en l'affranchissant du bien et du mal par la violence : « En effet, j'arrache le masque à sa figure traîtresse et pleine de boue, et je fais tomber un à un, comme des boules d'ivoire sur un bassin d'argent, les mensonges sublimes avec lesquels il se trompe lui-même.» (II, p. 59). C'est finalement bien une philosophie nietzschéenne que l'on peut pressentir dans ces lignes, car Les Chants de Maldoror se veulent une vision poétique qui prône le crépuscule des idoles; sa méchanceté n'est que la face visible d'une violence instinctive et irrépressible qui naît de l'oppression constante d'une âme torturée. La lutte permanente qui est menée contre l'humanité et le divin vise à briser la finitude de la morale, mais aussi les mensonges que les hommes construisent pour eux-mêmes, afin, dans l'horizon d'un humanisme sadique et vicié, désaxé et désemparé après les maux du siècle, de libérer l'homme et de trouver sa place dans un nouvel ordre qui soit fait à son image. Il ne s'agit pas d'une image qui enfouisse une partie de l'homme pour n'exprimer que ce qui est honorable, mais pour faire valoir une nouvelle conception de l'homme qui soit entière et qui embrasse tout ce qui le constitue, sans oblitérer la pulsion, la violence, le vice et le crime. En cela, Les Chants de Maldoror ne sont pas le récit déconstruit des élucubrations d'un hommedémon insensé, mais l'épopée d'une humanité qui se redécouvre en un grand cri rageur et vindicatif. 
Nous achèverons en évoquant ces mots de Gaston Bachelard dans son Lautréamont: "Nous comprenons donc bien que certaines âmes se détournent de Lautréamont. Mais Lautréamont est ainsi. Il illustre un complexe net entre tous, un complexe dangereux, terrible, fortement névrosant» (1995, p. 39). Cette névrose, pour le lecteur, c'est celle qui naît en contemplant son reflet dans le miroir ; non à titre personnel, mais en tant qu'être humain, c'est-à-dire inventeur et unique acteur de la méchanceté. Maldoror incarne la méchanceté : ce n'en est pas une figure figée, comme celle de Satan, mais il est l'essence du mal, un mal qui transcende la morale, un mal absurde, un mal humain. Et comme tout geste absurde, il a un sens profond; car Les Chants de Maldoror sont le cri d'une liberté qui se cherche, le cri rageur d'une liberté qui ne se réalise que dans la méchanceté, et tout le paradoxe névrosant de cette œuvre est qu'elle propose une croisade émouvante contre la méchanceté humaine, mais une croisade menée par l'être le plus malveillant qui soit. Et cette croisade ne trouve son achèvement que dans une libération violente, pour un apaisement qui se situe par-delà le bien et le mal.

\section{Bibliographie}

BACHElard, Gaston. (1995), Lautréamont, Paris, José Corti.

DuCASSE, Isidore. (1973), CEuvres complètes, Paris, Gallimard, coll. « Poésie Gallimard ». 


\title{
Résumé
}

Si la littérature française a donné naissance à bien des figures de méchants, celle créée par Lautréamont, dans Les Chants de Maldoror, demeure une des plus mystérieuses et une des plus extrêmes. Ce personnage se révèle incarner un sadisme et une cruauté difficiles à appréhender tant ils sont imaginatifs ; cependant, les animalisations et métamorphoses de Maldoror soulignent une volonté de stigmatiser l'humanité et ses exactions, tandis que son attitude révoltée et blasphématoire se veut une remise en question du manichéisme moral biblique tout en suggérant un horizon de liberté pour l'humanité une fois délivrée du bien et du mal.

\begin{abstract}
French litterature gave rise to many cruel characters; Lautreamont's eponym protagonist in Les Chants de Maldoror may be one of the most cryptic and extreme. This character personifies sadism and cruelty beyond understanding; however metamorphosis and metaphorical construction underline the intention to stigmatize mankind and human acts of violence, whereas Maldoror's rebellious and blasphemous behaviour is meant to question moral and biblical Manichaeism while suggesting a new horizon of freedom for humanity once released from good and evil.
\end{abstract}

